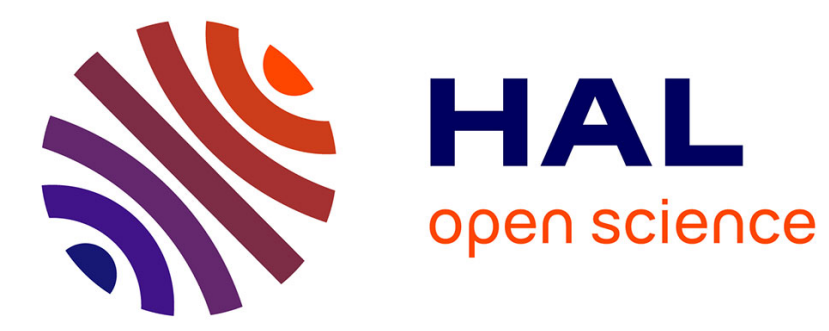

\title{
Series containing squared central binomial coefficients and alternating harmonic numbers \\ John Campbell
}

\section{To cite this version:}

John Campbell. Series containing squared central binomial coefficients and alternating harmonic numbers. 2018. hal-01836014

\section{HAL Id: hal-01836014 \\ https://hal.science/hal-01836014}

Preprint submitted on 11 Jul 2018

HAL is a multi-disciplinary open access archive for the deposit and dissemination of scientific research documents, whether they are published or not. The documents may come from teaching and research institutions in France or abroad, or from public or private research centers.
L'archive ouverte pluridisciplinaire HAL, est destinée au dépôt et à la diffusion de documents scientifiques de niveau recherche, publiés ou non, émanant des établissements d'enseignement et de recherche français ou étrangers, des laboratoires publics ou privés. 


\title{
Series containing squared central binomial coefficients and alternating harmonic num- bers
}

\author{
John M. Campbell
}

\begin{abstract}
We introduce an integration method for evaluating infinite series involving alternating harmonic numbers that generalizes many of the results in [Integral Transforms Spec Funct 28:7, 2017]. Using this method, we provide new evaluations for series containing factors of the form $\left(\begin{array}{c}2 n \\ n\end{array}\right)^{2} H_{2 n}^{\prime}$ that cannot be evaluated using known generating functions involving harmonic-type numbers. A closed-form evaluation is given for the series
\end{abstract}

$$
\sum_{n=1}^{\infty}\left(-\frac{1}{16}\right)^{n} \frac{\left(\begin{array}{c}
2 n \\
n
\end{array}\right)^{2} H_{2 n}^{\prime}}{n+1}
$$

and we describe why the problem of symbolically computing this particular series is especially difficult. The integration technique given in our article may be applied to evaluate natural generalizations and variants of the above series, such as the binomial-harmonic series

$$
\sum_{n=1}^{\infty} \frac{\left(-\frac{1}{16}\right)^{n}\left(\begin{array}{c}
2 n \\
n
\end{array}\right)^{2} H_{2 n}^{\prime}}{2 n-1}=\frac{(\pi-4 \ln (2)) \Gamma^{2}\left(\frac{1}{4}\right)}{8 \sqrt{2} \pi^{3 / 2}}-\frac{\sqrt{\frac{\pi}{2}}(\pi+4 \ln (2)-4)}{\Gamma^{2}\left(\frac{1}{4}\right)}
$$

introduced in our article. Our integration-based method, when applied in conjunction with the main lemma from [Ramanujan $\mathrm{J} 46: 2,2018$ ], also provides a new way of evaluating series involving even-indexed harmonic numbers.

Mathematics Subject Classification (2010). Primary 33C75, 33C20; Secondary $65 \mathrm{~B} 10$.

Keywords. Alternating harmonic number, Infinite series, Integral transform, Harmonic series. 


\section{Introduction}

The symbolic computation of infinite series containing harmonic numbers is a fascinating subject that traces back to Euler's seminal work on the evaluation of series of the form $\sum_{n=1}^{\infty} \frac{H_{n}}{n^{m}}$. Of course, the study of the closedform evaluation of infinite series with summands containing factors given by harmonic-type numbers remains an active area of research. In our present article, we introduce a useful technique for evaluating infinite series involving alternating harmonic numbers.

New techniques for computing series containing products of harmoniclike numbers and central binomial coefficients are given in $[1,2,3,4,5,6,7$, $8,9,10,11,14]$. Letting $G$ denote Catalan's constant, our article is inspired in part by the recent formula

$$
\frac{4 G-12 \ln 2+6}{\pi}=\sum_{n=1}^{\infty} \frac{\left(\begin{array}{c}
2 n \\
n
\end{array}\right)^{2}}{16^{n}(2 n-1)^{2}} H_{2 n}
$$

that was proved in [5] following two separate techniques, through an application of the operator $T_{\ln \text {,arcsin }}$ introduced in [5] and also through the use of a generating function given by Chen in 2016 in [7], in which a variety of generating functions involving harmonic-like numbers and binomial-type expressions are given. The interesting formula given in (1.1) was subsequently noted in [13], in which the evaluation of series of the form

$$
\sum_{n=1}^{\infty} \frac{H_{p n}^{(m)}}{n\left(\begin{array}{c}
n+k \\
k
\end{array}\right)}
$$

is explored. From the elegant evaluation provided in (1.1), together with the technique introduced in [3] for evaluating series containing $\left(\begin{array}{c}2 n \\ n\end{array}\right)^{2} H_{n}$ as a factor for indices $n \in \mathbb{N}$, we are inspired to explore the symbolic computation of series with summands involving factors such as $\left(\begin{array}{c}2 n \\ n\end{array}\right)^{2} H_{2 n}$ and

$$
\left(\begin{array}{c}
2 n \\
n
\end{array}\right)^{2} H_{2 n}^{\prime}
$$

letting $H_{m}^{\prime}$ denote the alternating harmonic number of order $m \in \mathbb{N}_{0}$, with $H_{2 n}^{\prime}=H_{2 n}-H_{n}$.

The integral transform $T_{\text {ln,arcsin }}$ introduced in [5] in 2017 is used to prove new evaluations for series involving factors of the form indicated in (1.2), such as the series

$$
\sum_{n \in \mathbb{N}}\left(\frac{\left(\begin{array}{c}
2 n \\
n
\end{array}\right)}{4^{n}(n+1)(2 n-1)(2 n-3)}\right)^{2} H_{2 n}^{\prime}
$$

introduced in [5]. In our present article, we introduce an integration-based method that may be applied to summations of the form

$$
\sum_{n \in \mathbb{N}}\left(\begin{array}{c}
2 n \\
n
\end{array}\right)^{2} H_{2 n}^{\prime} \cdot g_{n}
$$


for sequences $\left(g_{n}: n \in \mathbb{N}\right)$, with this new technique generalizing and building upon a number of the results from [5]. Since the integration method from [3] is used to compute series of the form

$$
\sum_{n \in \mathbb{N}}\left(\begin{array}{c}
2 n \\
n
\end{array}\right)^{2} H_{n} \cdot g_{n}
$$

we thus obtain a new approach toward the problem of evaluating series involving even-indexed harmonic numbers.

In [2], it is noted that there appears to be an interesting connection between series involving $\left(\frac{1}{32}\right)^{n}\left(\begin{array}{c}2 n \\ n\end{array}\right)^{2} H_{n}$ and series containing factors of the form

$$
\left(-\frac{1}{16}\right)^{n}\left(\begin{array}{c}
2 n \\
n
\end{array}\right)^{2} H_{2 n}^{\prime}
$$

It is noted in [2] that the integration method from [3] cannot be applied directly to evaluate the series

$$
\sum_{n=1}^{\infty}\left(\frac{1}{32}\right)^{n} \frac{\left(\begin{array}{c}
2 n \\
n
\end{array}\right)^{2} H_{n}}{2 n-3}
$$

in the sense that it is unclear how the required integral expression given by this method may be evaluated. If the Mathematica function Simplify is applied to this expression, we obtain a complicated expression involving numerous parameter derivatives of ${ }_{3} \tilde{F}_{2}(-1)$ functions. A re-indexing argument is used in [2] to evaluate (1.5), and this is used, together with the expression for $(1.5)$ in terms of parametric derivatives on ${ }_{3} \tilde{F}_{2}(-1)$ mappings, to show that the alternating series

$$
\sum_{n=1}^{\infty}\left(-\frac{1}{16}\right)^{n}\left(\begin{array}{c}
2 n \\
n
\end{array}\right)^{2} \frac{(2 n+3) H_{2 n}^{\prime}}{(2 n-1)(n+1)}
$$

is equal to

$$
-\frac{2}{3}+\frac{5(\pi-4 \ln (2)) \Gamma^{2}\left(\frac{1}{4}\right)}{12 \sqrt{2} \pi^{3 / 2}}-\frac{\sqrt{2 \pi}(-20+3 \pi+12 \ln (2))}{3 \Gamma^{2}\left(\frac{1}{4}\right)},
$$

which motivates the study of summations containing (1.4) as a factor. We introduce an interesting integration technique in our present article to evaluate series of this form, offering something of a partial solution to an open problem given in [2].

As noted in [2], it is surprising that the fundamental lemma from [3] can be used to evaluate (1.6), since this lemma is specifically "designed" for series containing $H_{n}$ for $n \in \mathbb{N}$, as opposed to series involving evenindexed alternating harmonic numbers. The evaluation of generalizations and variations of (1.6) is left as an open problem in [2], and serves as a basis for our present article. 
This article is also inspired by the formula

$$
\frac{6 \ln (2)-2}{\pi}=\sum_{n=1}^{\infty} \frac{\left(\begin{array}{c}
2 n \\
n
\end{array}\right)^{2} H_{2 n}}{16^{n}(2 n-1)}
$$

which was recently proved in [4] through the use of Fourier-Legendre theory. In [4], it is noted that from the FL expansion

$$
-\frac{3}{2}+\sum_{n=1}^{\infty}\left(\frac{(-1)^{n}}{2}\left(\frac{1}{n}-\frac{1}{n+1}\right)-\left(\frac{1}{n}+\frac{1}{n+1}\right)\right) P_{n}(2 x-1)
$$

for the expression $\ln (1-\sqrt{x})$, together with the formula

$$
\int_{0}^{1} x^{n} \ln (1-\sqrt{x}) d x=-\frac{H_{2 n+2}}{n+1},
$$

we may evaluate the series in (1.7), by using the formula

$$
\int_{0}^{1} \mathbf{E}(\sqrt{x}) \ln (1-\sqrt{x}) d x=-\frac{\pi}{2} \sum_{n=0}^{\infty} \frac{\left(\begin{array}{c}
2 n \\
n
\end{array}\right)^{2} H_{2 n+2}}{16^{n}(n+1)(1-2 n)}
$$

given in [4] and applying the FL series provided in (1.8). It is not clear how one would go about proving new FL expansion formulas in order to evaluate more general classes of harmonic-binomial sums of the form noted in (1.3). This inspires us to construct a simple integration technique that easily allows us to evaluate series of this form, by analogy with the fundamental lemma from [3], which is given below. From something of a heuristic perspective, this lemma shows us that if we apply the binomial expansion for $\sqrt{1-x^{2}}$ and then multiply the Maclaurin series for this elementary function by the inverse of this mapping, we can manipulate the resultant equation so as to be able to express a given harmonic-binomial series in terms of a relatively manageable integral and the underlying non-harmonic summation of the initial series.

Lemma 1.1. If the series

$$
\sum_{n=0}^{\infty}(-1)^{n}\left(\begin{array}{c}
\frac{1}{2} \\
n
\end{array}\right) f(n) \frac{x^{2 n} \ln \left(1-x^{2}\right)}{\sqrt{1-x^{2}}}
$$

is integrable on $[0,1]$ for a sequence $\left(f_{n}\right)_{n \in \mathbb{N}_{0}}$, and if we let $g_{n}=\frac{f_{n}}{16^{n}(2 n-1)}$, then

$$
\sum_{n=0}^{\infty} g(n)\left(\begin{array}{c}
2 n \\
n
\end{array}\right)^{2} H_{n}
$$

equals the product of $\frac{2}{\pi}$ with the sum of

$$
\int_{0}^{1}\left(\sum_{n=0}^{\infty}(-1)^{n}\left(\begin{array}{c}
\frac{1}{2} \\
n
\end{array}\right) f(n) \frac{x^{2 n} \ln \left(1-x^{2}\right)}{\sqrt{1-x^{2}}}\right) d x
$$

and $\pi \ln (2) \sum_{n=0}^{\infty} \frac{\left(\begin{array}{c}2 n \\ n\end{array}\right)^{2} f(n)}{16^{n}(1-2 n)}[3]$. 
As discussed in [3], the above lemma provides us with a useful way of producing new Ramanujan-like series for $\frac{1}{\pi}$ that cannot feasibly be proved using the generating functions from papers such as [1].

\section{Main results}

The following lemma may be regarded as a direct analogue of the main technique from [3].

Lemma 2.1. If we let $\left(f_{n}: n \in \mathbb{N}_{0}\right)$ denote a sequence such that

$$
\sum_{n=0}^{\infty}(-1)^{n} x^{2 n} \sqrt{1-x^{2}}\left(\begin{array}{c}
-\frac{1}{2} \\
n
\end{array}\right) f_{n} \ln (x)
$$

is integrable on $[0,1]$, writing $g_{n}$ in place of $\frac{f_{n}}{n+1}$, we have that the series

$$
\sum_{n \in \mathbb{N}_{0}}\left(\frac{1}{16}\right)^{n} H_{2 n}^{\prime}\left(\begin{array}{c}
2 n \\
n
\end{array}\right)^{2} g_{n}
$$

is equal to $\frac{4}{\pi}$ times the definite integral with respect to $x$ of $(2.1)$ over $[0,1]$ plus

$$
\frac{1}{2} \sum_{n=0}^{\infty}\left(\frac{1}{16}\right)^{n} \frac{\left(\begin{array}{c}
2 n \\
n
\end{array}\right)^{2}(2 \ln (2)(n+1)+1)}{(n+1)^{2}} f_{n} .
$$

Proof. This follows immediately from the identity whereby

$$
\int_{0}^{1} x^{2 n} \sqrt{1-x^{2}} \ln (x) d x
$$

evaluates as

$$
\frac{\sqrt{\pi}\left(H_{n-\frac{1}{2}}-H_{n+1}\right) \Gamma\left(n+\frac{1}{2}\right)}{8 \Gamma(n+2)}
$$

for $\Re(n)>-\frac{1}{2}$.

\subsection{A seemingly recalcitrant series containing alternating harmonic num- bers}

As described below, the problem of evaluating the infinite series given in (2.2) below is very difficult, and gives rise to challenging new problems related to the subject matter from $[2,3,5]$. The problem of determining the symbolic value of the infinite series in Question 2.2 below is inspired in part by the evaluation (1.1) from [5] and the equation (1.7) introduced in [4]. If we apply partial fraction decomposition in the summand in (1.6), we obtain the series given below.

Question 2.2. How can the series

$$
\sum_{n=0}^{\infty}\left(-\frac{1}{16}\right)^{n} \frac{\left(\begin{array}{c}
2 n \\
n
\end{array}\right)^{2} H_{2 n}^{\prime}}{n+1}
$$

be evaluated in closed form? 
Since there are known algorithms for computing series of the forms noted below for $z \in \mathbb{Z}_{>0}[2,3]$, it seems natural to consider the evaluation of variants of the following series, further motivating the problem given in Question 2.2.

$$
\begin{array}{ll}
\sum_{n=1}^{\infty} \frac{\left(\begin{array}{c}
2 n \\
n
\end{array}\right)^{2} H_{n}}{16^{n}(n+z)} & \sum_{n=1}^{\infty} \frac{\left(\begin{array}{c}
2 n \\
n
\end{array}\right)^{2} H_{n}}{16^{n}(n+z)^{2}} \\
\sum_{n=1}^{\infty} \frac{\left(\begin{array}{c}
2 n \\
n
\end{array}\right)^{2} H_{n}}{16^{n}(2 n-2 z+1)} & \sum_{n=1}^{\infty} \frac{\left(\begin{array}{c}
2 n \\
n
\end{array}\right)^{2} H_{n}}{16^{n}(2 n-2 z+1)^{2}} \\
\sum_{n=1}^{\infty} \frac{\left(\begin{array}{c}
2 n \\
n
\end{array}\right)^{2} H_{n}}{32^{n}(n+z)} & \sum_{n=1}^{\infty} \frac{\left(\begin{array}{c}
2 n \\
n
\end{array}\right)^{2} H_{n}}{32^{n}(n+z)^{2}} \\
\sum_{n=1}^{\infty} \frac{\left(\begin{array}{c}
2 n \\
n
\end{array}\right)^{2} H_{n}}{32^{n}(2 n-2 z+1)} & \sum_{n=1}^{\infty} \frac{\left(\begin{array}{c}
2 n \\
n
\end{array}\right)^{2} H_{n}}{32^{n}(2 n-2 z+1)^{2}}
\end{array}
$$

Mathematica 11 is not able to evaluate the equivalent formulation

$$
\sum_{n=0}^{\infty}\left(-\frac{1}{16}\right)^{n} \frac{\left(\begin{array}{c}
2 n \\
n
\end{array}\right)^{2}\left(H_{2 n}-H_{n}\right)}{n+1}
$$

of the series in Question 2.2 at all. We remark that it took Mathematica 11 about two straight hours of running time before providing any output after inputting the expression in (2.3), and only outputted the same symbolic form in (2.3), without providing any kind of simplification or evaluation. This illustrates that the problem of evaluating the series in Question 2.2 is highly nontrivial. Unsurprisingly, Maple 18 cannot evaluate the series in (2.3) at all. Even if we directly make use of the definition of the term alternating harmonic number in our attempts to evaluate (2.2) using computer algebra systems, we find that Mathematica 11 cannot evaluate

$$
\sum_{n=0}^{\infty}\left(-\frac{1}{16}\right)^{n} \frac{\left(\begin{array}{c}
2 n \\
n
\end{array}\right)^{2} \sum_{i=1}^{2 n} \frac{(-1)^{i+1}}{i}}{n+1}
$$

in that Mathematica 11 is only able to to compute (2.4) as

$$
\sum_{n=0}^{\infty}\left(-\frac{1}{16}\right)^{n} \frac{\left(\begin{array}{c}
2 n \\
n
\end{array}\right)^{2}(\ln (2)-\Phi(-1,1,2 n+1))}{n+1},
$$

with this evaluation following trivially from the definition of the Lerch transcendent. Maple 18 is only able to provide the redundant evaluation

$$
\sum_{n=0}^{\infty}\left(-\frac{1}{16}\right)^{n} \frac{\left(\begin{array}{c}
2 n \\
n
\end{array}\right)^{2}\left(\frac{1}{2} \Psi\left(n+\frac{1}{2}\right)-\frac{1}{2} \Psi(n+1)+\ln (2)\right)}{n+1}
$$

of (2.4), letting $\Psi$ denote the digamma function.

Now that we have discussed how current CAS software cannot evaluate (2.2) directly, we proceed to elaborate on the following claim concerning the 
difficulty of solving Question 2.2: It is not feasible to apply the generating functions from $[1,7]$ to evaluate the series in $(2.2)$.

In [7], Chen noted that the power series

$$
\sum_{n=1}^{\infty} \frac{\left(\begin{array}{c}
2 n \\
n
\end{array}\right) H_{2 n}^{\prime}}{n+1} x^{n}
$$

may be evaluated as

$$
\frac{1}{2 x}\left((1-\sqrt{1-4 x})+(1+\sqrt{1-4 x}) \ln \left(\frac{1+\sqrt{1-4 x}}{2}\right)\right),
$$

and we remark that Mathematica is able to compute (2.5) directly. However, there is no known symbolic evaluation for

$$
\sum_{n=1}^{\infty} \frac{\left(\begin{array}{c}
2 n \\
n
\end{array}\right)^{2} H_{2 n}^{\prime}}{n+1} x^{n}
$$

In terms of power series that are known to have closed-form evaluations, it appears that (2.5) is by far the "closest" or most similar to (2.2), modulo scalar multiples of the variables involved. There are a number of binomialharmonic generating functions that are much less similar to (2.2) computed in $[1,7]$, including the generating functions noted below.

$$
\begin{array}{ll}
\left.G\left(\begin{array}{c}
2 n \\
n
\end{array}\right) H_{n} ; x\right) & G\left(\left(\begin{array}{c}
2 n \\
n
\end{array}\right) H_{2 n} ; x\right) \\
G\left(\frac{\left(\begin{array}{c}
2 n \\
n
\end{array}\right)}{n+1} H_{n} ; x\right) & G\left(\frac{\left(\begin{array}{c}
2 n \\
n
\end{array}\right)}{n}\left(H_{2 n-1}-H_{n}\right) ; x\right)
\end{array}
$$

From the evaluation of the ordinary generating function

$$
G\left(\frac{\left(\begin{array}{c}
2 n \\
n
\end{array}\right) H_{2 n}^{\prime}}{n+1} ; x\right)
$$

given in (2.6), we find that

$$
\sum_{n=1}^{\infty}\left(-\frac{1}{16}\right)^{n} \frac{\left(\begin{array}{c}
2 n \\
n
\end{array}\right)\left(H_{2 n}-H_{n}\right)}{n+1} x^{n}
$$

is equal to

$$
\frac{4\left(\sqrt{x+4}-(\sqrt{x+4}+2) \ln \left(\frac{1}{4}(\sqrt{x+4}+2)\right)-2\right)}{x}
$$

From the above discussion, we see that

$$
G\left(\left(-\frac{1}{16}\right)^{n} \frac{\left(\begin{array}{c}
2 n \\
n
\end{array}\right)\left(H_{2 n}-H_{n}\right)}{n+1} ; x\right)
$$

is the "closest" possible generating function relative to (2.2) that can be computed in terms of elementary functions. So, given the relatively simple closed-form evaluation given in (2.7), it would seem as if the summation in Question 2.2 could be evaluated by making use of an integral formula for 
central binomial coefficients. As discussed below, it appears that this would not be feasible, which is interesting since many similar series can be computed directly through the use of Wallis-type integrals.

A standard version of Wallis's integral formula is given by the identity whereby

$$
\left(\begin{array}{c}
2 n \\
n
\end{array}\right)=\int_{0}^{\frac{\pi}{2}} \frac{2(2 \cos (t))^{2 n}}{\pi} d t
$$

for $n \in \mathbb{N}_{0}$. So, we find that the problem of evaluating the series in Question 2.2 is equivalent to the problem of evaluating

$$
\begin{aligned}
& 2-\frac{4 \sqrt{2} \Gamma\left(\frac{3}{4}\right)^{2}}{\pi^{3 / 2}}- \\
& \frac{4}{\pi} \int_{0}^{\frac{\pi}{2}} \sqrt{1+\cos ^{2}(t)} \ln \left(\frac{1+\sqrt{1+\cos ^{2}(t)}}{2}\right) \sec ^{2}(t) d t
\end{aligned}
$$

which cannot be evaluated using Mathematica 11 or Maple 18. It is unclear as to how the above integral may be evaluated, especially since the two terms resulting from the expansion of the integrand in

$$
\int_{0}^{\frac{\pi}{2}} \sqrt{1+\cos ^{2}(t)}\left(\ln \left(1+\sqrt{1+\cos ^{2}(t)}\right)-\ln (2)\right) \sec ^{2}(t) d t
$$

cannot be separately integrated on $\left[0, \frac{\pi}{2}\right]$. So, we see that the problem of evaluating the series in Question 2.2 is equivalent to determining a closedfrom for the difficult integral

$$
\int_{1}^{2} \frac{\sqrt{u} \ln \left(\frac{1+\sqrt{u}}{2}\right)}{\sqrt{2-u}(u-1)^{3 / 2}} d u,
$$

which cannot be evaluated by Mathematica 11 or Maple 18. The above integral cannot be evaluated by rewriting the expression $\ln \left(\frac{1+\sqrt{u}}{2}\right)$ as $\ln (1+$ $\sqrt{u})-\ln (2)$ and expanding the resultant integrand, since the integral

$$
\int_{1}^{2} \frac{\sqrt{u}}{\sqrt{2-u}(u-1)^{3 / 2}} d u
$$

does not converge. It is not at all obvious as to how to evaluate the definite integral displayed in (2.8). It seems as if it would not be feasible to expand a given combination of factors in the integrand in (2.8) as a Taylor series and integrate term-by-term. For example, we have that

$$
\frac{1}{\sqrt{2-u}(u-1)^{3 / 2}}=\sum_{n=0}^{\infty}(-1)^{n} 2^{n+2}\left(u-\frac{3}{2}\right)^{n}\left(\begin{array}{c}
\frac{1}{2}(n+((n-1) \bmod 2)) \\
\frac{1}{2}
\end{array}\right),
$$

but it seems that this expansion cannot be directly used to evaluate (2.8), especially since state-of-the-art computer algebra systems cannot evaluate

$$
\int_{1}^{2}\left(u-\frac{3}{2}\right)^{n} \sqrt{u} \ln \left(\frac{1+\sqrt{u}}{2}\right) d u
$$

for a parameter $n \in \mathbb{N}_{0}$. 
If we try to make use of the standard integral formula for the Catalan sequence whereby

$$
\frac{\left(\begin{array}{c}
2 n \\
n
\end{array}\right)}{n+1}=\int_{0}^{4} \frac{x^{n} \sqrt{\frac{4-x}{x}}}{2 \pi} d x
$$

we see that the problem of evaluating the summation in Question 2.2 is equivalent to the evaluation of

$$
\int_{0}^{4}-\frac{\sqrt{\frac{4-x}{x}} \ln \left(\frac{1}{4}(\sqrt{x+4}+2)\right)}{\pi \sqrt{x+4}} d x
$$

which cannot be evaluated by Mathematica 11 or Maple 18. Equivalently, this summation is equal to

$$
-\frac{2}{\pi} \int_{0}^{1} \frac{\sqrt{\frac{1-x}{x}} \ln (1+\sqrt{1+x})}{\sqrt{1+x}} d x-\frac{4 \sqrt{2 \pi} \ln (2)}{\Gamma\left(\frac{1}{4}\right)^{2}}+\frac{\Gamma\left(\frac{1}{4}\right)^{2} \ln (2)}{\sqrt{2} \pi^{3 / 2}},
$$

with state-of-the-art computer algebra systems being unable to evaluate the above integral. The Maclaurin series for $\ln (1+y)$ cannot be used to evaluate the above integral, as may be verified. Also, the Maclaurin series for $\sqrt{\frac{1-x}{1+x}}$ cannot be used to compute this integral.

Even if we try to make use of non-standard integral formulas for central binomial coefficients or Catalan-type sequences as a way of approaching Question 2.2, we obtain recalcitrant integrals. For example, if we try to apply the recently-discovered Catalan number formula

$$
C_{n}=\frac{1}{\pi} \int_{0}^{\infty} \frac{\sqrt{t}}{\left(t+\frac{1}{4}\right)^{n+2}} d t
$$

given in [12], we find that (2.2) is equal to

$$
\int_{0}^{\infty}-\frac{8 \sqrt{2} \sqrt{x} \ln \left(\frac{1}{2}\left(\sqrt{\frac{1}{4 x+1}+1}+1\right)\right)}{\pi \sqrt{2 x+1}(4 x+1)^{3 / 2}} d x
$$

with Mathematica 11 and Maple 18 unable to compute the above expression.

If we try to make use of the standard integral formula for the factor $\frac{1}{n+1}$ in the summand in (2.2), we find that this would require the evaluation of

$$
\sum_{n=0}^{\infty}\left(-\frac{1}{16}\right)^{n}\left(\begin{array}{c}
2 n \\
n
\end{array}\right)^{2}\left(H_{2 n}-H_{n}\right) x^{n}
$$

with Mathematica 11 and Maple 18 being unable to evaluate (2.10) at all.

From the above discussion, it should be clear that we cannot use known generating functions for harmonic/binomial-type sequence to evaluate the seemingly recalcitrant series in (2.2). Moreover, it seems that it is not feasible to use known integral formulas for alternating harmonic numbers to evaluate 
the infinite series in (2.2). For example, if we try to make use of the known identity

$$
\int_{0}^{1} \ln (x+1) x^{2 n} d x=\frac{-1+2 \ln 2+4 n \ln 2}{(2 n+1)^{2}}-\frac{H_{2 n}^{\prime}}{2 n+1}
$$

noted in [5], we find that the problem of symbolically computing the aforementioned series is equivalent to the problem of evaluating the difficult integral

$$
\int_{0}^{1} \frac{\ln (x+1)(\mathbf{E}(i x)-\mathbf{K}(i x))}{x^{2}} d x
$$

which cannot be evaluated by Mathematica 11 or Maple 18.

Question 2.2 is also interesting because it is unclear as to how the corresponding series

$$
\sum_{n=0}^{\infty}\left(-\frac{1}{16}\right)^{n} \frac{\left(\begin{array}{c}
2 n \\
n
\end{array}\right)^{2} H_{2 n}}{n+1}
$$

with non-alternating harmonic numbers of even index could be computed symbolically, as a corollary of the following theorem, using the main lemma from [3].

Theorem 2.3. The series

$$
\sum_{n=0}^{\infty}\left(-\frac{1}{16}\right)^{n} \frac{\left(\begin{array}{c}
2 n \\
n
\end{array}\right)^{2} H_{2 n}^{\prime}}{n+1}
$$

is equal to

$$
2+\frac{(4 \ln (2)-\pi) \Gamma^{2}\left(\frac{1}{4}\right)}{4 \sqrt{2} \pi^{3 / 2}}-\frac{\sqrt{2 \pi}(4+\pi+4 \ln (2))}{\Gamma^{2}\left(\frac{1}{4}\right)} .
$$

Proof. This follws immediately from Lemma 2.1 by letting $f_{n}=(-1)^{n}$.

It is truly remarkable that the integration technique given by Lemma 2.1 provides such a simple way of evaluating the infinite series in $(2.2)$, since the problem of determining the symbolic value of the series in Theorem 2.2 may be regarded as being very difficult, as described above.

\subsection{Generalizations and variants}

Through the use of Lemma 2.1, we also obtain the following extensions of the above theorem.

$$
\begin{aligned}
& \sum_{n=0}^{\infty}\left(-\frac{1}{16}\right)^{n} \frac{\left(\begin{array}{c}
2 n \\
n
\end{array}\right)^{2} H_{2 n}^{\prime}}{n+2}= \\
& -\frac{8}{9}+\frac{\sqrt{2 \pi}(4+\pi+4 \ln (2))}{3 \Gamma^{2}\left(\frac{1}{4}\right)}+\frac{(4(4+3 \ln (2))-3 \pi) \Gamma^{2}\left(\frac{1}{4}\right)}{108 \sqrt{2} \pi^{3 / 2}} \\
& \sum_{n=0}^{\infty}\left(-\frac{1}{16}\right)^{n} \frac{\left(\begin{array}{c}
2 n \\
n
\end{array}\right)^{2} H_{2 n}^{\prime}}{n+3}=
\end{aligned}
$$




$$
\begin{aligned}
& \frac{128}{225}-\frac{(32+39 \pi-156 \ln (2)) \Gamma^{2}\left(\frac{1}{4}\right)}{540 \sqrt{2} \pi^{3 / 2}}-\frac{\sqrt{2 \pi}(316+95 \pi+380 \ln (2))}{375 \Gamma\left(\frac{1}{4}\right)^{2}} \\
& \sum_{n=0}^{\infty}\left(-\frac{1}{16}\right)^{n} \frac{\left(\begin{array}{c}
2 n \\
n
\end{array}\right)^{2} H_{2 n}^{\prime}}{n+4}= \\
& \frac{\sqrt{2 \pi}(516+145 \pi+580 \ln (2))}{875 \Gamma^{2}\left(\frac{1}{4}\right)}+\frac{(1360-273 \pi+1092 \ln (2)) \Gamma^{2}\left(\frac{1}{4}\right)}{20580 \sqrt{2} \pi^{3 / 2}}-\frac{512}{1225}
\end{aligned}
$$

Applying partial fraction decomposition to the rational component of the series in (1.6), we obtain the expression

$$
\frac{1}{3} \sum_{n=1}^{\infty}\left(\frac{1}{16}\right)^{n} \frac{(-1)^{n+1}\left(\begin{array}{c}
2 n \\
n
\end{array}\right)^{2} H_{2 n}^{\prime}}{n+1}+\frac{8}{3} \sum_{n=1}^{\infty}\left(\frac{1}{16}\right)^{n} \frac{(-1)^{n}\left(\begin{array}{c}
2 n \\
n
\end{array}\right)^{2} H_{2 n}^{\prime}}{2 n-1}
$$

and in view of Theorem 2.3 this strongly motivates the evaluation of the series given in the following theorem, since much of our present article is inspired by the open problem given in [2].

Theorem 2.4. The equality

$$
\sum_{n=0}^{\infty} \frac{\left(-\frac{1}{16}\right)^{n}\left(\begin{array}{c}
2 n \\
n
\end{array}\right)^{2} H_{2 n}^{\prime}}{2 n-1}=\frac{(\pi-4 \ln (2)) \Gamma^{2}\left(\frac{1}{4}\right)}{8 \sqrt{2} \pi^{3 / 2}}-\frac{\sqrt{\frac{\pi}{2}}(\pi+4 \ln (2)-4)}{\Gamma^{2}\left(\frac{1}{4}\right)}
$$

holds.

Proof. This follows immediately from Lemma 2.1 by letting $f_{n}=\frac{(-1)^{n}(n+1)}{2 n-1}$.

The fundamental lemma introduced in our present article my also be used to evaluate

$$
\sum_{n=0}^{\infty}\left(-\frac{1}{16}\right)^{n} \frac{\left(\begin{array}{c}
2 n \\
n
\end{array}\right)^{2} H_{2 n}^{\prime}}{2 n-2 z-1}
$$

for $z \in \mathbb{Z}_{>0}$, yielding the following evaluations.

$$
\begin{aligned}
& \sum_{n=0}^{\infty}\left(-\frac{1}{16}\right)^{n} \frac{\left(\begin{array}{c}
2 n \\
n
\end{array}\right)^{2} H_{2 n}^{\prime}}{2 n-3}= \\
& \frac{\sqrt{2 \pi}(-4+\pi+4 \ln (2))}{6 \Gamma^{2}\left(\frac{1}{4}\right)}+\frac{\Gamma^{2}\left(\frac{1}{4}\right)(16+3 \pi-12 \ln (2))}{216 \sqrt{2} \pi^{3 / 2}} \\
& \sum_{n=0}^{\infty}\left(-\frac{1}{16}\right)^{n} \frac{\left(\begin{array}{c}
2 n \\
n
\end{array}\right)^{2} H_{2 n}^{\prime}}{2 n-5} \\
& \frac{(39 \pi-4(8+39 \ln (2))) \Gamma^{2}\left(\frac{1}{4}\right)}{1080 \sqrt{2} \pi^{3 / 2}}-\frac{\sqrt{\frac{\pi}{2}}(-316+95 \pi+380 \ln (2))}{375 \Gamma^{2}\left(\frac{1}{4}\right)} \\
& \sum_{n=0}^{\infty}\left(-\frac{1}{16}\right)^{n} \frac{H_{2 n}^{\prime}\left(\begin{array}{c}
2 n \\
n
\end{array}\right)}{2 n-7}=
\end{aligned}
$$




$$
\frac{(1360+273 \pi-1092 \ln (2)) \Gamma^{2}\left(\frac{1}{4}\right)}{41160 \sqrt{2} \pi^{3 / 2}}+\frac{\sqrt{\frac{\pi}{2}}(-516+145 \pi+580 \ln (2))}{875 \Gamma^{2}\left(\frac{1}{4}\right)}
$$

\subsection{On the evaluation of series involving even-indexed harmonic numbers and squared central binomial coefficients}

As a way of further demonstrating the utility of Lemma 2.1, we offer a simplified proof of the following result that had been introduced in [4].

Theorem 2.5. $\sum_{n=1}^{\infty}\left(\frac{1}{16}\right)^{n} \frac{\left(\begin{array}{c}2 n \\ n\end{array}\right)^{2} H_{2 n}}{2 n-1}=\frac{6 \ln (2)-2}{\pi}[4]$.

Proof. Letting $f_{n}=\frac{n+1}{2 n-1}$, by Lemma 2.1 we have that

$$
\sum_{n=1}^{\infty}\left(\frac{1}{16}\right)^{n} \frac{\left(\begin{array}{c}
2 n \\
n
\end{array}\right)^{2} H_{2 n}^{\prime}}{2 n-1}=\frac{2-2 \ln (2)}{\pi}
$$

and from the evaluation of

$$
\sum_{n=1}^{\infty}\left(\frac{1}{16}\right)^{n} \frac{\left(\begin{array}{c}
2 n \\
n
\end{array}\right)^{2} H_{n}}{2 n-1}
$$

given in [3] we obtain the desired result.

Remark 2.6. It is interesting to note that Mathematica is able to evaluate the series in Theorem 2.5 as

$$
\frac{1}{2}{ }_{2} F_{1}{ }^{(0,0,1,0)}\left[\begin{array}{c|c}
-\frac{1}{2}, \frac{1}{2} & 1 \\
1 & 1
\end{array}\right]+\frac{2 \ln (2)}{\pi}
$$

and since

$$
\sum_{n=1}^{\infty} \frac{\left(\begin{array}{c}
2 n \\
n
\end{array}\right)^{2} H_{n}}{16^{n}(2 n-1)}={ }_{2} F_{1}(0,0,1,0)\left[\begin{array}{c|c}
-\frac{1}{2}, \frac{1}{2} & 1 \\
1 &
\end{array}\right],
$$

as noted in [3] we thus obtain another proof of the above theorem.

Mimicking the proof of Theorem 2.5 given above, by letting $f_{n}=$ $\frac{n+1}{2 n-(2 z-1)}$ for natural numbers $z$, we obtain the evaluations indicated below.

$$
\begin{aligned}
& \frac{90 \ln (2)-46}{27 \pi}=\sum_{n=1}^{\infty}\left(\frac{1}{16}\right)^{n} \frac{\left(\begin{array}{c}
2 n \\
n
\end{array}\right)^{2} H_{2 n}}{2 n-3} \\
& \frac{8010 \ln (2)-4814}{3375 \pi}=\sum_{n=1}^{\infty}\left(\frac{1}{16}\right)^{n} \frac{\left(\begin{array}{c}
2 n \\
n
\end{array}\right)^{2} H_{2 n}}{2 n-5} \\
& \frac{80010 \ln (2)-52894}{42875 \pi}=\sum_{n=1}^{\infty}\left(\frac{1}{16}\right)^{n} \frac{\left(\begin{array}{c}
2 n \\
n
\end{array}\right)^{2} H_{2 n}}{2 n-7}
\end{aligned}
$$

The series in (1.1) from [5] is a natural extension of Chen's series for $\frac{1}{\pi}$ from [7], and it is natural to explore new methods of deriving the formula in (1.1), in the hope of arriving at similar results. 
Theorem 2.7. The series

$$
\sum_{n=1}^{\infty}\left(\frac{1}{16}\right)^{n} \frac{\left(\begin{array}{c}
2 n \\
n
\end{array}\right)^{2} H_{2 n}}{(2 n-1)^{2}}
$$

is equal to $\frac{6+4 G-12 \ln (2)}{\pi}[5]$.

Proof. This follows from Lemma 2.1 by letting $f_{n}=\frac{n+1}{(2 n-1)^{2}}$, using the symbolic form for

$$
\sum_{n=1}^{\infty}\left(\frac{1}{16}\right)^{n} \frac{\left(\begin{array}{c}
2 n \\
n
\end{array}\right)^{2} H_{n}}{(2 n-1)^{2}}
$$

given in [3].

Remark 2.8. By rewriting the expression $H_{2 n}$ in the summand in (2.13)

$$
H_{2 n}=\int_{0}^{1} \frac{1-x^{2 n}}{1-x} d x
$$

and reversing the order of summation and integration, it can be shown that the above theorem also follows from Theorem 2.5.

Using the technique given in the proof of Theorem 2.7, we obtain the following results.

$$
\begin{aligned}
& \frac{16 G+36-44 \ln (2)}{9 \pi}=\sum_{n=1}^{\infty}\left(\frac{\left(\begin{array}{c}
2 n \\
n
\end{array}\right)}{4^{n}(2 n-3)}\right)^{2} H_{2 n} \\
& \frac{19200 G+50132-50820 \ln (2)}{16875 \pi}=\sum_{n=1}^{\infty}\left(\frac{\left(\begin{array}{c}
2 n \\
n
\end{array}\right)}{4^{n}(2 n-5)}\right)^{2} H_{2 n} \\
& \frac{11289600 G+32183036-29233260 \ln (2)}{13505625 \pi}=\sum_{n=1}^{\infty}\left(\frac{\left(\begin{array}{c}
2 n \\
n
\end{array}\right)}{4^{n}(2 n-7)}\right)^{2} H_{2 n}
\end{aligned}
$$

Again through an application of Lemma 2.1 together with the fundamental lemma from [3] as in the proof of Theorem 2.7, we obtain the following evaluations, none of which can be symbolically computed by Mathematica 11 or Maple 18.

$$
\begin{aligned}
& \frac{92+24 \pi-180 \ln (2)}{27 \pi}=\sum_{n=1}^{\infty}\left(\frac{1}{16}\right)^{n} \frac{\left(\begin{array}{c}
2 n \\
n
\end{array}\right)^{2} H_{2 n}}{n+2} \\
& \frac{9628+1920 \pi-16020 \ln (2)}{3375 \pi}=\sum_{n=1}^{\infty}\left(\frac{1}{16}\right)^{n} \frac{\left(\begin{array}{c}
2 n \\
n
\end{array}\right)^{2} H_{2 n}}{n+3} \\
& \frac{105788+17920 \pi-160020 \ln (2)}{42875 \pi}=\sum_{n=1}^{\infty}\left(\frac{1}{16}\right)^{n} \frac{\left(\begin{array}{c}
2 n \\
n
\end{array}\right)^{2} H_{2 n}}{n+4}
\end{aligned}
$$


It is interesting to note that the main lemma from [3] cannot be applied directly to evaluate series of the form

$$
\sum_{n=0}^{\infty}\left(\frac{1}{16}\right)^{n} \frac{\left(\begin{array}{c}
2 n \\
n
\end{array}\right)^{2} H_{2 n}}{(n+z)^{2}}
$$

for $z \in \mathbb{Z}$. A general method for evaluating series of this form is given in [2], through the use of a clever re-indexing argument.

Theorem 2.9. The infinite series

$$
\sum_{n=1}^{\infty} \frac{\left(\begin{array}{c}
2 n \\
n
\end{array}\right)^{2} H_{2 n}}{16^{n}(n+1)^{2}}
$$

is equal to $\frac{16 G+24-48 \ln (2)}{\pi}+4-8 \ln (2)$.

Proof. Through a direct application of Lemma 2.1 we find that the series

$$
\sum_{n=1}^{\infty} \frac{\left(H_{2 n}-H_{n}\right)\left(\begin{array}{c}
2 n \\
n
\end{array}\right)^{2}}{16^{n}(n+1)^{2}}
$$

is equal to

$$
\frac{1}{2}{ }_{4} F_{3}\left[\begin{array}{c|c}
\frac{1}{2}, \frac{1}{2}, 1,1 & 1 \\
2,2,2 & 1
\end{array}\right]-4+\frac{16 \ln (2)}{\pi} .
$$

So, from the evaluation

$$
\sum_{n=1}^{\infty} \frac{\left(\begin{array}{c}
2 n \\
n
\end{array}\right)^{2} H_{n}}{16^{n}(n+1)^{2}}=16+\frac{32 G-64 \ln (2)}{\pi}-16 \ln (2)
$$

introduced in [2], we find that the problem of evaluating binomial-harmonic series in the above theorem reduces to the evaluation of a ${ }_{4} F_{3}(1)$ series with parameters in $\frac{1}{2} \mathbb{Z}$. However, the expression

$$
{ }_{4} F_{3}\left[\begin{array}{c|c}
\frac{1}{2}, \frac{1}{2}, 1,1 & 1 \\
2,2,2 & 1
\end{array}\right]
$$

cannot be evaluated by Mathematica, not even through the use of

\section{FunctionExpand}

or through the use of the equivalent hypergeometric form in

$$
\sum_{n=0}^{\infty} \frac{\left(\begin{array}{c}
2 n \\
n
\end{array}\right)^{2}}{16^{n}(n+1)^{3}}
$$

which Maple is only able to evaluate in terms of the Barnes G-function as

$$
\frac{1}{\pi} \cdot G_{4,4}^{4,1}\left(\begin{array}{l|l}
-1 & \begin{array}{l}
1,2,2,2 \\
1,1, \frac{1}{2}, \frac{1}{2}
\end{array}
\end{array}\right)
$$




$$
\text { Series containing }\left(\begin{array}{c}
2 n \\
n
\end{array}\right)^{2} H_{2 n}^{\prime}
$$

However, if we make use of the canonical integral formula

$$
\frac{\left(\begin{array}{c}
2 i \\
i
\end{array}\right)}{i+1}=\int_{0}^{4} \frac{x^{i} \sqrt{\frac{4-x}{x}}}{2 \pi} d x
$$

for the Catalan sequence, we observe that the problem of finding a symbolc evaluation for the hypergeometric expression in (2.16) is equivalent to evaluating the seemingly recalcitrant integral

$$
\frac{4}{\pi} \int_{0}^{4} \frac{x-4+2 \sqrt{4-x}(1-2 \ln (2)+\ln (2+\sqrt{4-x}))}{x^{3 / 2}} d x
$$

which Mathematica, interestingly, is able to directly evaluate, as

$$
\frac{16(-2 G+3+\pi(\ln (2)-1))}{\pi},
$$

thus providing us with the desired result.

Based on the evaluation provided in the above theorem, through the use of iterative re-indexing, we obtain the following new evaluations.

$$
\begin{aligned}
& \frac{64 G+144-176 \ln (2)}{9 \pi}-\frac{8}{27}-\frac{32 \ln (2)}{9} \\
& =\sum_{n=1}^{\infty}\left(\frac{\left(\begin{array}{c}
2 n \\
n
\end{array}\right)}{4^{n}(n+2)}\right)^{2} H_{2 n} \\
& \frac{76800 G+200528-203280 \ln (2)}{16875 \pi}-\frac{2752}{3375}-\frac{512 \ln (2)}{225} \\
& =\sum_{n=1}^{\infty}\left(\frac{\left(\begin{array}{c}
2 n \\
n
\end{array}\right)}{4^{n}(n+3)}\right)^{2} H_{2 n} \\
& \frac{45158400 G+128732144-116933040 \ln (2)}{13505625 \pi}-\frac{115456}{128625}-\frac{2048 \ln (2)}{1225} \\
& =\sum_{n=1}^{\infty}\left(\frac{\left(\begin{array}{c}
2 n \\
n
\end{array}\right)}{4^{n}(n+4)}\right)^{2} H_{2 n}
\end{aligned}
$$

The integral transform introduced in our present article, together with the evaluations presented above, nicely generalize some of the concepts presented in [5]. Letting $C_{n}$ denote the Catalan number of order $n$, we see that the formula

$$
\frac{1500 G-1946+2048 \ln 2}{3375 \pi}-\frac{244}{3375}+\frac{8 \ln 2}{225}=\sum_{n=1}^{\infty} \frac{C_{n}^{2}}{16^{n}(2 n-3)^{2}(2 n-1)^{2}} H_{2 n}^{\prime}
$$

introduced in [5] follows from some of the main results put forth in the present section of our article together with some of the main results from [3], by rewriting the above summand as

$$
\frac{\left(\begin{array}{c}
2 n \\
n
\end{array}\right)^{2}}{16^{n}(n+1)^{2}(2 n-3)^{2}(2 n-1)^{2}} H_{2 n}
$$


and applying partial fraction decomposition to the rational component

$$
\frac{1}{(n+1)^{2}(2 n-3)^{2}(2 n-1)^{2}}
$$

in the above summand.

\section{Conclusion}

The main lemma introduced in our article can also be applied to series that do not involved squared central binomial coefficients. For example, a direct application of Lemma 2.1 shows that

$$
\sum_{n=1}^{\infty} \frac{2^{-6 n} H_{2 n}\left(\begin{array}{c}
2 n \\
n
\end{array}\right)\left(\begin{array}{c}
4 n \\
2 n
\end{array}\right)}{n+1}
$$

is equal to

$$
\frac{8}{3}+\frac{8 \sqrt{2}-40 \sqrt{2} \ln (2)+8 \ln (1+\sqrt{2})}{3 \pi},
$$

and this can also be determined using Chen's generating function for

$$
\left(\frac{\left(\begin{array}{c}
2 n \\
n
\end{array}\right)}{n+1} H_{2 n}: n \in \mathbb{N}_{0}\right)
$$

given in [7], together with a Wallis-type integral. The exploration of further applications of Lemma 2.1 seems like a worthwhile area to pursue.

\section{References}

[1] Boyadzhiev, K.N.: Series with central binomial coefficients, Catalan numbers, and harmonic numbers. J. Integer Seq. 15(1), Article 12.1.7, 11 (2012)

[2] Campbell, J.M.: New series involving harmonic numbers and squared central binomial coefficients. HAL e-prints hal-01774708 (2018)

[3] Campbell, J.M.: Ramanujan-like series for $\frac{1}{\pi}$ involving harmonic numbers. Ramanujan J. 46, 373-387 (2018)

[4] Campbell, J.M., D'Aurizio, J., Sondow, J.: On the interplay between hypergeometric functions, complete elliptic integrals and Fourier-Legendre series expansions. ArXiv e-prints arXiv:1710.03221v1 (2017)

[5] Campbell, J.M., Sofo, A.: An integral transform related to series involving alternating harmonic numbers. Integral Transforms Spec. Funct. 28, 547-559 (2017)

[6] Cantarini, M., D'Aurizio, J.: On the interplay between hypergeometric series, Fourier-Legendre expansions and Euler sums. ArXiv e-prints arXiv:1806.08411 (2018)

[7] Chen, H.: Interesting series associated with central binomial coefficients, Catalan numbers and harmonic numbers. J. Integer Seq. 19(1), Article 16.1.5, 11 (2016)

[8] Chu, W., Zheng, D.: Infinite series with harmonic numbers and central binomial coefficients. Int. J. Number Theory 5(3), 429-448 (2009)

[9] Guillera, J.: More hypergeometric identities related to Ramanujan-type series. Ramanujan J. 32(1), 5-22 (2013) 
[10] Liu, H., Zhou, W., Ding, S.: Generalized harmonic number summation formulae via hypergeometric series and digamma functions. J. Difference Equ. Appl. 23(7), 1204-1218 (2017)

[11] Nicholson, M.: Quadratic Transformations of Hypergeometric Function and Series with Harmonic Numbers. ArXiv e-prints arXiv:1801.02428 (2018)

[12] Qi, F., Shi, X.-T., Liu, F.-F. (2015). An integral representation, complete monotonicity, and inequalities of the Catalan numbers. ResearchGate Techinical Report. doi: 10.13140/RG.2.1.3754.4806.

[13] Sofo, A.: General order Euler sums with multiple argument. J. Number Theory 189, 255-271 (2018)

[14] Wan, J.G.f.: Random Walks, Elliptic Integrals and Related Constants. Ph.D., University of Newcastle (2013)

John M. Campbell

York University

4700 Keele St

Toronto, ON

M3J 1P3

e-mail: jmaxwellcampbell@gmail.com 